\title{
FAIRNESS IN GAMES: A STUDY ON CHILDREN'S AND ADULTS' UNDERSTANDING OF PROBABILITY
}

\author{
RITA BATISTA \\ Universidade Federal de Pernambuco, Brasil \\ rica.basil@gmail.com \\ RUTE BORBA \\ Universidade Federal de Pernambuco, Brasil \\ resrborba@gmail.com \\ ANA HENRIQUES \\ Instituto de Educação, Universidade de Lisboa, Portugal \\ achenriques@ie.ulisboa.pt
}

\begin{abstract}
This study aims to analyze the reasoning that children and adults with the same school level use to assess and justify the fairness of games, considering aspects of probability such as randomness, sample space, and comparison of probabilities. Data collection included a Piagetian clinical interview based on games of chance. The results showed participants' judgments about the fairness of the games depended mainly on the understanding of independence of events, analysis of the sample space, and perception of proportionality when comparing probabilities, and indicated they may have misunderstandings about these ideas. The similar low performance of adults and children on probabilistic reasoning tasks, indicates that the maturity and experience of the adults were not enough to develop appropriate probabilistic reasoning and to instrumentalize it to assess the fairness of a game consistently. Thus, teaching interventions to expand and consolidate students' learning in the field of probability are recommended and the activities presented in this study may serve as a basis for such interventions.
\end{abstract}

Keywords: Statistics education research; Probability; Randomness; Sample space; Fairness in games; Children and adult students

\section{INTRODUCTION}

Probabilistic knowledge has acquired great relevance in mathematics education since it plays an instrumental role in learning diverse curricular topics, and it is required to make correct interpretations of stochastic data and informed decisions in daily life situations involving uncertainty and inferences (Batanero et al., 2016; Sharma, 2016). The presence of probability in the mathematics curriculum is advocated to prepare students for a conscious exercise of citizenship in contemporary times, as both adults and children face difficulties in this area of knowledge (e.g., Bryant \& Nunes, 2012). In this sense, the literature (Batanero et al., 2016; Bryant \& Nunes, 2012) and curriculum guidelines (ME, 2017; Franklin et al., 2007; NCTM, 2000) suggest teaching probability from the early school years, to develop students' probabilistic reasoning in a processual and continuous way, starting from an intuitive basis at the lowest levels to formalisation at the highest levels.

In Brazil, the current curriculum guidelines document (BNCC; ME, 2017), introduces the teaching of probability from the 1st year of elementary education ( 6 to 10 years of age). The objective is to:

... promote the understanding that not all phenomena are deterministic. In consequence, the beginning of the work in probability is focused on the development of the randomness notion, so that students understand that there are certain, impossible and probable events.... In this phase, it is important that students verbalize, in situations involving chance, the results that could occur as opposed to what actually happened, initiating the construction of the sample space. (p. 274) 
Although the teaching of probability has become part of the mathematics curriculum of elementary education in many countries, including Brazil, its teaching has been neglected both because teachers do not feel prepared to teach probability and also because of the absense of the topic in textbooks (Campos \& Pietropaolo, 2013). Jones and Tarr (2007) argued the probabilistic mistakes made by adults are indicative of a lack of previous education. Moreover, Chiesi and Primi (2009) found that college students showed more fragile knowledge of probability than children, even when both groups had access to the teaching of probability, and justified that "formal conceptions, sometimes, have no lasting effect, and some biases could even arise from them" (p. 161). Children, however, have primary intuitions about probability that result from beliefs and everyday experiences, which are independent of formal education but may influence the classroom practices and, thus, to expand and deepen their probabilistic understanding, intervention through teaching is essential (Fischbein, 1987). In this context, where the teaching and learning of probability is configured in a complex and challenging process to create learning contexts for the development of students' probabilistic reasoning (Bryant \& Nunes, 2012) and still a poorly researched area, it is necessary to study effective approaches to promote students' understanding of various concepts associated with probability and help them develop normative intuitions and overcome the cognitive difficulties they face in that learning.

According to Cañizares et al. (2004), an effective approach to teaching and learning probability is the use of chance games and experiments involving coins or dice, which may help students understand the concepts of chance and independent and mutually exclusive events, as well as observe random events, becoming aware of uncertainty and the need to make probabilistic estimates. These perspectives emphasize the importance of assessing students' understanding and intuitive beliefs about probability, including their ideas that relate chance games and fairness, to help in developing effective teaching approaches aiming to promote and broaden their probabilistic knowledge. It is also pertinent to understand to what extent maturity and experience influence the probabilistic understanding of students, by analyzing the similarities and differences between children and adults with similar school level.

Reflecting on these concerns, this paper intends to contribute to research in the field of probabilistic education by clarifying the relations that children and adults establish among fairness in games and necessary elements to understand probability (Bryant \& Nunes, 2012), and whether maturity influences this understanding. While the main aim of the paper is to analyze the reasoning children and adults (with the same school level) use to evaluate and justify the fairness of games, the activities that support the study reported in this paper may also serve as a basis for planning teaching interventions to promote the understanding of probability of children and adults.

\section{BACKGROUND ON FAIRNESS IN GAMES}

Although some studies in the literature focus on fairness in games, few studies compare the understanding of children and adults in this theme, except the research of Watson and Moritz (2003). A study by Pratt (2000) used a micro world in a computer game for participants to assess the behavior of objects (dice, coins), intending to verify the knowledge about randomness of children aged 10 to 11 years. The results of this study showed children related randomness to four central characteristics: lack of stability, irregularity, unpredictability, and fairness. Paparistodemou, et al. (2002) conducted a survey to analyze how children aged 6 to 8 years could organize balls of an electronic game (micro world) to match the teams' chances of winning, that is, to make the game fair considering the sample space. In this game, children could control the number, size, and position of the teams' balls, but they had no influence on the randomly bouncing white ball. The children associated a fair organization of the game with i) casual movement (lack of pattern), ii) complex movement, iii) symmetry in the positioning of the teams' objects, and iv) equality in the sizes of the objects.

Cañizares et al. (2004) analyzed the conceptions of 10- and 14- year old students about fair games in two situations: one in a context of equal chances for the players and the other with unequal chances, but with the possibility of compensatory prizes to make the game fair. The authors concluded the majority of the students believe that fair game is synonymous of equiprobable results and, therefore, their difficulties were not in judging whether the game was fair or not, but in determining whether the outcomes were equiprobable. In general, the results improved with age and the mathematical ability of the students. Regarding children's reasoning about fairness in games, the researchers classified the conceptions into two categories that may or may not involve the equiprobability bias. Thus, they 
identified students with difficulties using proportional reasoning, students associating fair play with the use of the same elements (cards, balls), children who considered balance or proportionality as a form of fairness, students who managed to change the prize pool to make the game fair, and students who only considered the game to be fair when there was equiprobability, regardless the possibility of compensatory balance through unequal awards.

A study by Vidakovic et al. (1998) with eighth grade students sought to examine ideas and beliefs about fairness in the context of games, considering chance and the sample space. The results showed that most of the students' ideas came from their everyday lives and that only part of their conclusions were based on the observations and experiments carried out during the study. Students considered a game fair when everyone had the same chances of winning, although some students argued the numbers 1,2 , and 3 would be more likely to occur than the numbers 4,5 and 6 and that a fairer game would "mix" or "spread" numbers like 2, 4 and 6 for one player and 1, 3 and 5 for another. Lidster et al. (1996) carried out studies with children from the third, fifth, seventh, and ninth grades. In one of the studies, the researchers used three dice: blue (biased towards number 2), red (not modified), and blue and white (with numbers 1, 2 and 3 duplicated), and the participants were asked if the dice were fair. Younger children emphasized an iconic belief related to personal experiences, myths, or anthropomorphism, as well as arguments based on the physical characteristics of the dice. Older students suggested systematic trials to assess fairness of the dice. A dichotomous belief that the dice could sometimes be fair and other times unfair was also observed.

Longitudinal research on students' beliefs about dice fairness was described by Watson and Moritz (2003). Two studies focused on beliefs and strategies for judging fairness were carried out, one with students from the third, fifth, seventh, and ninth grades and the other with some of these students after 3 or 4 years. In this study, the same resources (dice) from Lidster et al. (1996) were used, adding a green die with characteristics like the blue one. The results showed progress over time for many of the students who initially had beliefs or strategies that fell into the most elementary level: iconic, the belief that certain numbers are favoured based on personal experiences. Even so, some students maintained idiosyncratic and contradictory beliefs (that there are numbers that come out more often, but everyone has the same chance) and others maintained the belief that the dice were always fair. Regarding the strategies, some students remained at the same level and a few of them were at a lower level. The authors, however, did not observe an association among beliefs about fairness and strategies for judging it.

\section{THEORETICAL FRAMEWORK}

\subsection{ESSENTIAL ELEMENTS FOR UNDERSTANDING PROBABILITY}

Bryant and Nunes (2012) considered probability a very complex concept involving understanding several elements they refered to as "cognitive demands", among which we highlight three main ones that support our study:

(i) Understanding randomness: understanding the nature, consequences and use of randomness in everyday life;

(ii) Working out the sample space: recognizing all possible events and sequences in which they may occur; and

(iii) Comparing and quantifying probabilities: understanding probability as a quantity based on proportions and that the solution is often based on proportional calculations or relations.

In addition, the authors considered these elements as articulated with each other and fundamental to ensure the understanding of probability, being necessary to solve most basic probabilistic problems. Understanding randomness is an element that guarantees understanding fairness in games, but it is often complex because even adults have difficulties on the independence of events, when mistakenly believe that sequences like 1, 2, 3, 4 and 5 are less likely to be drawn at random than 5, 7, 2, 9, 15. Recognizing the size of the sample space and the possible events of this set is, for these authors, one of the most important steps to solve probabilistic situations. Regarding comparing and quantifying probabilities of different sample spaces, the authors argued that the proportional nature involved in probability calculations explain difficulties for children and adults. For example, although comparing the 
probability of getting even or odd in 12 balls numbered 1 to 12 only requires analysis of the elements of each event, comparing the probability of draw a blue ball in two boxes (Box A: 1 blue and 3 red balls and Box B: 2 blue and 5 red balls) requires a proportional reasoning.

It is worth noting that the curriculum trajectory indicated in the BNCC (ME, 2017) is in line with these basic elements of probability (Bryant \& Nunes, 2012), as it guides towards gradual learning that involves understanding of (i) randomness, which implies focus on uncertainty about results of experiments; (ii) sample space, which results in exploring the possibilities of the results of a random experiment; and (iii) quantifying and comparing of probabilities, which are translated into the proportional relations that consider the ratio between favourable cases and possible cases (classic view of probability).

\subsection{CONSTRUCTS OF PROBABILISTIC THINKING}

Jones et al. (1997) considered four constructs (sample space, probability of an event, comparison of probabilities, and conditional probability) to create a framework to identify the levels of children's probabilistic thinking. Later, Jones (2006) refined the conditional probability construct by emphasizing the independence of events. In each construct, four levels of probabilistic thinking were identified.

In Level 1 (Subjective), students adopt a narrow perspective in relation to situations of probability, not recognizing random phenomena and basing their judgments on subjective beliefs. They do not provide a complete list of the sample space, and in situations involving the probability of an event, comparison of probabilities, and conditional probabilities, they make subjective judgments, rather than quantitative, and assume that events are always related, not realizing the independence of events.

In Level 2 (Transitory), students build understandings but, in some situations, they can go back to Level 1, moving between subjective and quantitative judgments. In this level, they begin to distinguish between fair and unfair probability situations by comparing probabilities, and can identify a complete set of results for a simple experiment (one coin or die), but still have difficulties on more complex ones (e.g., three coins). They also may predict a more or less likely events based on quantitative judgments, but they can revert to subjective judgments. Understanding conditional probability eludes Level 2 students, but they show some recognition of consecutive events whether they are related.

At Level 3 (Informal quantitative), students use a systematic strategy when listing the results of a two-stage experiment and make quantitative judgments when determining the probability of an event and comparing probabilities, although the probabilities are not always expressed correctly. They begin to recognize that the probability of events changes in a non-replacement situation, although inaccurately differentiate independent and dependent events in "with" and "without" replacement situations.

In Level 4 (Numeric), students adopt strategies that allow systematic generation of the results of an experiment and assign and use numerical probabilities in (non)equitable situations. They use systematic strategies to list results of two and three stage experiments and use the sample space as a basis for finding and comparing numerical probabilities. This resource for numerical probability also operates on conditional probability, and the students' thinking at this level includes the recognition of conditional probability in relation to all the events of an experiment. They distinguish dependent and independent events in replacement and non-replacement situations, using numerical probabilities.

\section{METHODOLOGY}

\subsection{CONTEXT AND PARTICIPANTS}

This study is part of broader doctoral research about the understanding of children and adults (with the same school level) of fairness in games, considering cognitive demands of probability (Bryant \& Nunes, 2012). The study was conducted with 15 children and 15 adults who had not yet had access to formal probability content, from state schools of the Northeast of Brazil belonging to a community of low socioeconomic standard. The children were between 10 and 12 years old and studied at one school. The adults were between 25 and 65 years old and studied at two different schools in the same community. To select the participants, the study proposal was disclosed to the students of the classes and those who volunteered to participate were invited by the teacher to leave the room to be interviewed. The adults who participated in this study were part of a program called Youth and Adult Education in 
Brazil, which provided opportunities for young people and adults who had not completed their studies to return to school for new teaching and learning experiences. The adults had the same level of education as the participating children, that is, they attending 5th grade, which corresponded with the last year of the first stage of elementary school. No adult appeared to have more substantial experience with games of chance, only casual experiences with dice and coins, similar to the children. The adults, however, seemed to have a little more experience than children in bingo and lottery games.

\subsection{DATA COLLECTION AND INSTRUMENTATION}

For data collection, an audio recorded Piagetian clinical interview (Carraher, 1998) was conducted by the researcher (first author) and applied individually to all participating children and adults, in a calm and silent environment. The interviews were based on guiding questions that made it possible for the particpants to reflect on the responses and choices of the students about a proposed situation. In the interviews, six games and their fairness were explored, following the order they appear in Figure 1. Participants had the opportunity to handle the materials to become familiar with them before answering the interview questions and then analyzing the proposed situations to assess whether the games were fair or not, thereby, justifying their choices.

To explore each of the three elements of probability understanding that support our study (randomness, sample space, comparison of probabilities), two types of games were created: a fair and an unfair game. A fair game is one in which the involved participants have the same chances of winning, as defended by Borovenik (2016), who relied on Laplace hypothesis of equal probabilities for all elementary events as an approach that reflected fairness. Thus, it is necessary to have equiprobability in the results so each participant has equal conditions to win. In the analysis of games to assess fairness, the required participants' mental action will be based essentially on the comparison between the probabilities of winning by two or more players, within the presented contexts. To compare probabilities, it is also necessary to understand properties of randomness, such as independence of events, and to think about elements of the sample space present in games. Regarding independence of events, it is considered that each new test, each piece of lotto, bingo ball, face of a dice or balls of the box, keeps equal chances of being drawn and there is no internal or external influence that leads to one or the other result. Each trial is independent of the previous or the later and, thus, random sequences are created. Thus, given the connections among the three elements of probability understanding (Bryant \& Nunes, 2012) present in all games and their misunderstanding can produce misjudgments about fairness in games.

The games LOTO and BINGO focused especially on elements related to understanding randomness. Only the uncertainty about the results does not guarantee fairness in the game. So, the sequences present in the game cards, need to be random and the randomizers need to be honest. A random sequence is not characterized by its appearance, whether ordered or disordered and sequenced or without an apparent pattern, but how it was produced. Thus, if there is equiprobability in the drawing of numbers and the randomizers used to produce the sequence are honest, the sequence 1, 2, 3, 4 is as likely as $5,87,24,13$. Under these conditions, to assess fairness, participants need to understand when a sequence is random. For Bennett (1998), the impossibility of obtaining, in advance, desirable success in games of chance represents an important aspect of a random sequence: unpredictability. In this way, randomness ensures that there is no game scheme, rule, or formula to determine specific elements of the sequence, which is, thus, random. This condition is called fairness, or rather, fair play. The LOTO game can be considered a fair game, since each participant has an equal chance of winning, regardless of the numbers on the cards. The BINGO game, in turn, is considered unfair, as the participants' chances of winning are different, considering that the randomizers are not honest (some numbers are more likely to be drawn than others) and the cards benefit more one player than others. 


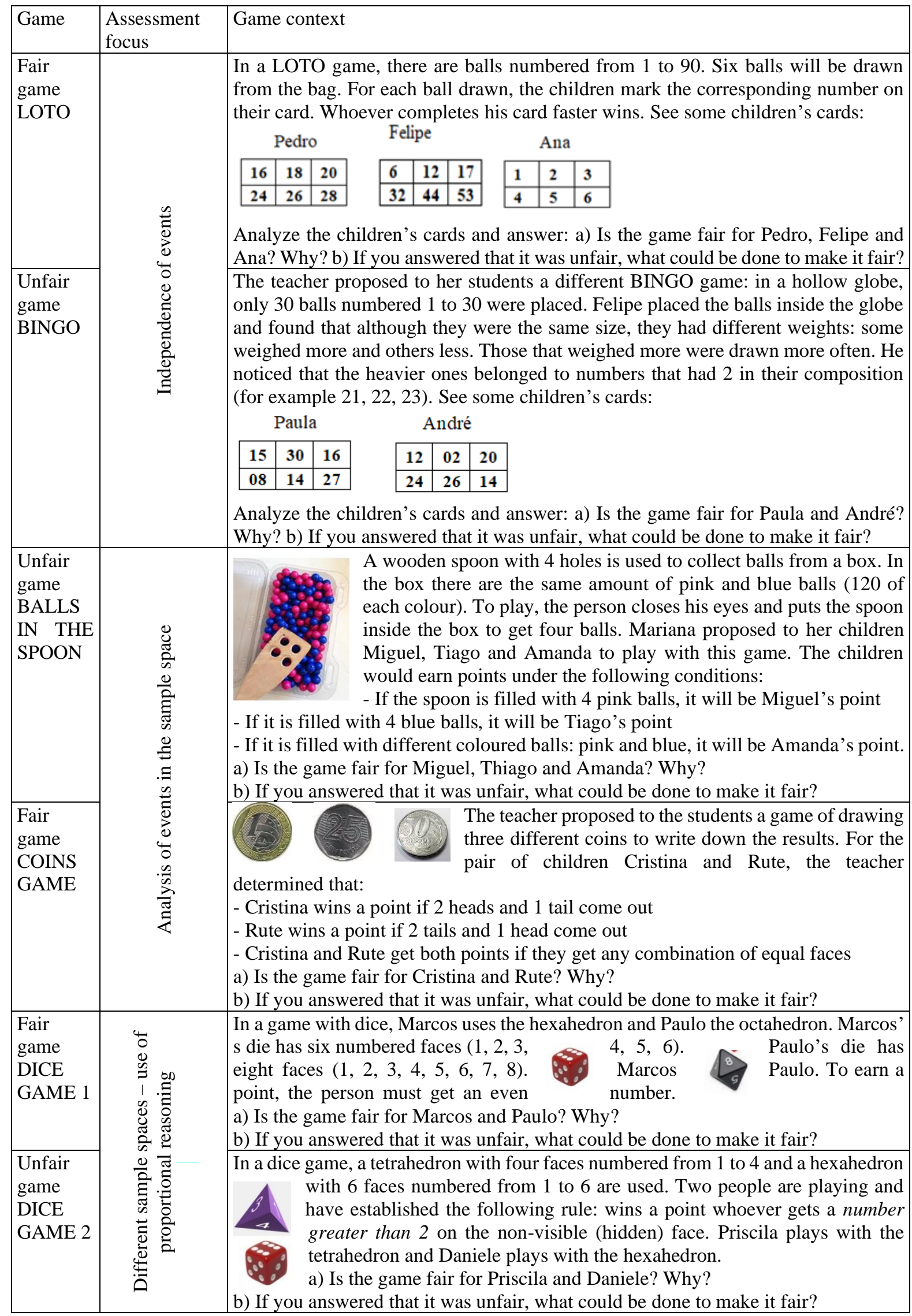

Figure 1. Games for clinical interview, considering randomness, sample space, comparisons of probability 
The BALLS IN THE SPOON and COINS games aim to verify whether the participants observe and relate the importance of the sample space to the comparison of probabilities. To assess whether the situation is fair, it is necessary for the participants to analyze the possibilities for each player, that is, check in the sample space the possibilities related to each event: "blue colour", "pink colour", or "blue and pink colours" in the case of the unfair game (BALLS IN THE SPOON) and "two heads and a tail" or "two tails and one head" in the fair game (COINS GAME). These games were not designed for students to make an exhaustive list of the sample space, but to reflect on the possibilities present in situations to assess fairness in games. For the unfair game BALLS IN THE SPOON, we used an activity inspired by Abrahamson (2006) referred in Bryant and Nunes (2012). In this game, there are different chances of the players winning due to the possibilities of organizing the balls in the spoon. In accordance with Figure 2, it is observed that for both Tiago and Miguel there is only one chance in 16 to obtain the same colour, while Amanda has 14 possibilities out of 16 to be successful in obtaining different colours. Thus, the game is unfair as there are far more chances for Amanda to win than for the other players.

\section{$88888888: 8888888$ 8888888888888888}

Figure 2. Possible results of the Game Balls in the spoon

In the two DICE games, the sample spaces were different, which required not only a comparison between the number of elements of each event, but a proportional relation between these elements and the sample space of each die. It was also necessary to realize that although the dice had a different format (tetrahedron, hexahedron and octahedron). The uncertainty about the results remained in equal proportion in each one of them. The DICE GAME 1, with the tetrahedron and hexahedron, was fair because there is the same probability for players to win, although the artefacts used were not the same. The comparison of probabilities occured in different sample spaces, so it was possible to use proportional reasoning to assess the situation. In this game the hexahedron had six faces, half of which have even numbers ( 3 in 6), as well as in the octahedron, which had four even and four odd faces (each 4 in 8). In DICE GAME 2, the tetrahedron had half of the faces larger than 2 (3 and 4) and the hexahedron had more than a half in this condition $(3,4,5$, and 6$)$, which made it an unfair game as the player with the hexahedron was more likely to win.

\subsection{DATA ANALYSIS}

The interview records of all participants were transcribed and translated into an analysis of their responses and choices about the proposed situations. This descriptive and interpretative data analysis (Wolcott, 2009), which was focused on participants' responses about the essential elements for understanding probability and fairness in games was based on the aspects of probability referred by Bryant and Nunes (2012) and the constructs and levels of probabilistic thinking of Jones et al. (1997) and Jones (2006). All the participants' responses were classified into three categories attending to the judgment of the game and the coherent justification: (i) correct answer, correctly reported that the game was fair or unfair and presented coherent arguments from a conceptual point of view; (ii) partially correct answer, if the judgment on fairness was correct but the justifications were not completely adequate conceptually, presenting partially correct information; and (iii) incorrect answer, when the student considered the game fair when it was unfair, or the opposite. Those who correctly reported the fairness of the game, but had no justifications or incorrect justification, also had their answers classified as incorrect. In complement, the levels of probabilistic thinking were also identified based on the previous analysis of coherent and mistaken arguments in participants' answers. 
In the following section we present the results of the analysis, evidenced with excerpts from the participants' answers (adults identified as A\# and children as C\#, to ensure their anonymity) that consider the diversity of responses obtained in the interviews.

\section{RESULTS}

Table 1 shows the results of children and adults' answers to all games, attending to their judgment about the fairness in games and the (in)coherent justification, involving the understanding of randomness, sample space and comparison of probabilities. Results show that the children performed better than the adults in all cases except the fair dice game. Moreover, all participants performed better on the unfair Bingo, fair Balls in the Spoon, and unfair Coins games and worse in the fair Loto and both fair and unfair Dice games.

Table 1. Results of the assessment made by children and adults about fair and unfair games

\begin{tabular}{lllll}
\hline Game & & $\begin{array}{l}\text { Correct } \\
\text { answer }\end{array}$ & $\begin{array}{l}\text { Partially } \\
\text { correct answer }\end{array}$ & $\begin{array}{l}\text { Incorrect } \\
\text { answer }\end{array}$ \\
\hline Fair game LOTO & Children & 3 & 3 & 9 \\
Unfair game BINGO & Adults* & 1 & 3 & 10 \\
& Children & 12 & 0 & 3 \\
Fair game BALLS IN THE SPOON & Adults & 9 & 0 & 6 \\
Unfair game COINS GAME & Children & 11 & 0 & 4 \\
& Adults* & 8 & 0 & 6 \\
Fair game DICE GAME 1 & Children & 12 & 1 & 2 \\
& Adults* & 9 & 2 & 3 \\
Unfair game DICE GAME 2 & Children & 0 & 0 & 15 \\
& Adults* & 0 & 2 & 12 \\
& Children & 0 & 0 & 15 \\
\hline
\end{tabular}

* One adult did not answer the question

\subsection{LOTO GAMES}

The few participants who were able to judge this game as fair and present a correct justification for that judgment report that all players (Pedro, Felipe and Ana) would have the same chances to win, as they had equal conditions to have their sequences of numbers drawn. For example:

C5 Yes. Because it's a matter of luck, right? I think everyone has the same chance of winning because they have all these numbers here in the bag, right?

A2 It's fair. I think anyone can win. Whatever I get, if it doesn't work for Felipe, may work for Pedro. You must take a risk ... one has, the other doesn't. Removing the ball, any of these can win.

Answers from other participants were influenced by the apparent difference in the number sequences shown on the cards $(16,18,20,24,26,28 ; 6,12,27,32,44,53 ; 1,2,3,4,5,6)$. This was the case for 6 participants (three children and three adults) whose answers were partially correct. Despite claiming that the situation was fair due to the players having the same number (one) of cards and to the uncertainty present in the situation, a conclusion that concerns the rules of the game, they incorrectly believed cards with the highest values would come out with more chances. They state, for example:

C8 I think it's fair, because the three have a card. Felipe is more likely to win than the others because he has the highest numbers. Ana has less chance because her numbers are very low.

A11 It depends on the person's luck. It's a game. It is fair because it is a tie. But Ana has less chance because her number is low. 
In the incorrect answers, the observation of the (random) sequences of the cards was a central element to assess the fairness in the game, which was considered not equiprobable and, therefore, not random. These answers were often related to the misunderstanding of random sequences, or the equiprobability present in the situation, due to beliefs that each result of a random experiment is independent of the previous or the later, and that larger or smaller numbers are more likely to be selected in a random draw, leading to incorrect judgments that Felipe (with alternating numbers) would have more chances than Ana (with ordered sequence). This misunderstanding is even more evident in the adults' statements who believe that, when playing the lottery, disorderly are more likely results than ordered sequences:

C1 No. Ana's [numbers] are in a row. It is unfair to her because her numbers are lower and those of others are higher. Felipe is more likely to win since he has the highest numbers. This [card] is the easiest to win because the little ones are more difficult to draw.

A1 I don't think so. Because here it is in sequence and here it is not in sequence. If they were in sequence, it would be 16, 17, 18, 19, 20, 21 ... I think it's not fair, because they gave more small numbers for Ana. She may even win... In any game that I think the number doesn't come out often in a row... it does not come out. They play differently. It is very rare, for example, come out 44,45 and 46 . When it is drawn like this, only two numbers are drawn in a row.

In accordance with the levels of probabilistic thinking and concerning to the "independence of events" construct, Level 1 (Subjective) relates to the students predisposition to judge consecutive events as always related and to the use of subjective reasoning that prevents understanding about the independence of events, and Level 2 (Transitory) shows some recognition about consecutive events. In this game, most of the students were at the Subjective level, verified in their justifications, like the ones of $\mathrm{C} 2$, who stated that "the first numbers are more difficult to get", and of A6 that reported "Felipe has a better chance, he has the highest numbers, when you play like that, you just draw more high numbers." These arguments evidence an understanding that the events are related and do not come out or are less likely to be drawn and that they do not realize the impartiality of the chance laws by establishing some dependency relationships or form of connection among trials.

When asked about what could be done to turn the game initially judged as unfair into a fair game, the participants mobilized their understanding of a fair game and often sought equiprobability in the situation. Those who believed that the numbers in sequence were less likely to come out, they proposed to change Ana's card to include "more random" numbers, which means more mixed numbers. Some participants also suggested to distribute the smaller numbers of Ana to the other cards, balancing them.

\subsection{BINGO GAME}

The participants' correct answers evaluate this game as unfair, as there are randomizers with greater weight (balls that have the number 2 in their composition), which have more probability of being drawn when the globe is rotated, compromising the equiprobability of the results. They correctly observed that the cards of the players Paula $(15,30,16,8,14,27)$ and André $(12,2,20,24,26,14)$ had different quantities of numbers that include the 2 , and pointed out the condition "equal chances vs different chances" as essential to that assessment, and presented justifications such as:

C1 No. This one [André] is easier to win because he has 2, 12, 20, 24 and 26 and she almost doesn't have number 2, only has 27. It's not fair for Paula as she doesn't have many chances to win.

A12 No, because André will take advantage. The number 2 ball will always fall first, André's ball. The heaviest ball throws the lightest balls up. André has 5 and Paula has only one. She has no chance to win.

Unlike children, some adults presented incoherent arguments for the unfairness of the game, as is the case of A8 who stated "No. Because the balls come out with higher numbers, don't they? Here you have more advantage because the numbers are higher, it's more, right?" and A9 who argued "It's fair for Paula, because she has the highest numbers. It's not fair for André." Even with unfair randomisers, the analysis showed the students' believed that some numbers come out more than others, as if there were some relationships among them. 
The incorrect answers, considering the game fair, are based on beliefs that there is a possibility for both players to win, even though the advantage and the chances for a given player are evident, and that the cards had numbers that were on the globe and this condition was fair. This is evidenced in the statements:

C1 It's a matter of luck and there are numbers up to 30 and here there are no more than 30 . Here there are 15, 16, 8, 14 and 27 and 30, then he will not get other balls that are not in the globe. I think it is fair because of that.

A10 Even if he has more chance it is a fair game. She can also win, there is a possibility.

It was also observed that sometimes the children, and especially adults, presented non-mathematical arguments to justify their choices about the fairness of the games. Some examples are: "Each one does what he likes to do. It is fair for those who like it. For those who do not like, it is not" (A13); "I think Ana has more chance because she's smarter, but I don't know why" (A9); and "It is an interactive game, for education. It's for fun, it's fair. I think everyone has the same chance" (C13). These arguments fit the Subjective level of probabilistic thinking, their judgments are based on subjective beliefs, not recognizing random phenomena.

In this game, the suggestions to turn the game judged unfair into a fair game were to have similar cards for the players or to keep only balls with the same weight, removing the heavier ones. However, an adult chose to keep the game as unfair, revealing an assumption that all games must be "unfair" in the sense that there is only one winner, and if the game is "fair" there will be a tie, when arguing "Leave it like this, unfair. Whoever wins, wins. If you put it evenly, it will be a tie" (A6).

\subsection{BALLS IN THE SPOON}

In correctly assessing this unfair game, the huge difference in players' chances of winning due to quantitative gap (less than $7 \%$ for Tiago and Miguel and more than $87 \%$ for Amanda) seems to have been evident for many of the participants who presented consistent justifications using numeric arguments regarding the probability of the events:

C3 She has a better chance to win because everything is mixed here, there is not just one colour, but it has two colours. It won't come out everything pink nor everything blue, there may be some possibility, but I don't think so. Everything comes out more mixed. It's just for Amanda.

A12 Amanda takes advantage because she has 120 balls of each, right? There are 240 balls for Amanda. And there are only 120 for Miguel and 120 for Tiago.

Some of the participants who incorrectly considered the situation to be fair assumed the possibility of winning makes the game fair even though they were aware there would be different chances for the players to win. Others presented justifications external to its situation and not based in mathematical arguments or related to the game. This is noted in the statements:

C10 I think Amanda has the best chance of winning because she has 120 blue balls and 120 pink balls and she has blue and pink, but they can also win. It's fair.

A6 Each one here has his chance. It's not bad for any of the three. Amanda always has the most advantage, but it's fair.

A9 I think it's fair for Tiago, because Tiago is blue, right? I think more blue [balls] comes out, but I can't say why. Pink can come out too, but I think it's blue

A15 If you are playing, it is fair. Tiago has more advantage because all Tiago students are intelligent, smart.

It was also noticed that few students analyzed the events of the sample space. Although some glimpses of understanding were observed, especially when they justified their choices by listing some of these elements. For example, "Amanda is more likely to win because if she gets two blues and two pinks she wins, then 3 pinks and 1 blue, 3 blues and 1 pink can come out" (A1).

These participants arguments also fit the Subjective level of the constructs referring to the sample space, in which they were able to list an incomplete set with some elements of the sample space. This 
view is not conclusive, however, since there was no specific requirement to list the elements of the sample space in the data collection instrument. When asked how to make the unfair game fair, the participants proposed adding another colour for Amanda or exclude her from playing the game, or to consider coloured balls for everyone (rule change).

\subsection{COINS GAME}

The participants who correctly justified this game as fair, even without fully expressing the sample space, considered both players as having the same chances of winning since the number of these chances is balanced among players:

C1 It's fair for both because they have the same chance of winning. Two heads and one tail or two tails and one head, is the same chance.

A14 I think it is fair, yes, because everything is equal. It has two tails and a head for Ruth and two heads and a tail for Cristina. It's balanced.

It was also observed that participants who presented incorrect answers, did not present justifications indicating the set of possibilities (sample space) for the launch of three coins. They focused only on the possibilities "heads and tails" as if the activity was referred to the sample space in the launch of one coin. They often repeated the rule, as a way of showing the "balance" present in the situation, and justified, for example, that one face would be more likely to come out than the other, or that the acceptance of the rules and participation in the game would be a condition for justice:

C12 Cristina has more chance because her [face] is heads and this one is tail. The heads come out more times.

A15 If she is playing it is because it is fair. Rute has more advantage because she is smart.

Luck was also an element that supported their justifications when arguing there is no way to cheat because the result depends on luck, as reported by A5 who mentioned, "the chances are equal because there is luck, then there is no way to say who will win."

The justifications often established a relationship between the most and the least probable outcomes: "Amanda has more chances than ...", and "Rute has less chances ...." In this way, this game focus on the sample space also allowed to analyze the construct probability of an event. Regarding this construct, several participants were at the Transitory level, which predicts the event more or less probable based on quantitative judgments, but can revert to subjective judgments, since although some arguments used did not present support of a probabilistic nature, they support their statements in numerical arguments.

The participants' suggestions for changing the game previously considered unfair to make it fair were to replace coins by dice or change rules. These ideas evidence that the proposals rest in the desire to match the chances of winning among the players, and thus reinforce the concept about a fair game that is also present in other games: the one in which everyone has the same chances of winning.

\subsection{DICE GAMES}

The fact that the dice has different formats (tetrahedron, hexahedron and octahedron) proved to be very challenging for the participants, in the two games explored. In fair DICE GAME 1, the probability of getting an even number was the same for both the hexahedron and the octahedron. This situation could be analyzed using proportionality, without the need of quantifying probabilities, considering half, more than a half or less than a half. In the hexahedron, half of the numbers are even, so there is half the chance of getting one. The same occurs in the octahedron. Most participants' answers show misunderstanding of proportional reasoning to compare probabilities of distinct sample spaces and considered the game unfair, either due to the different number of dice faces or due to the dice format or the greater or lesser number of even numbers in the artefacts, as observed in the following statements:

C15 It is unfair because Paulo has more numbers, more even numbers.

A4 I think not because his dice goes to 8 and his goes to 6 . Paulo has the possibility to win more times because he has more numbers. 
Only two partially coherent arguments were identified:

A2 Yes, it is fair. Both are playing equal even numbers. For me, they are playing well because both are playing to get even numbers.

A11 It is fair because he has a dice and will play. It is difficult to say who has more chance because it is a dice and then it will swing, it falls 1 , falls 2 , falls 3 .

In DICE GAME 2, it is necessary to compare probabilities to analyze the proportionality present in the situation that involves two different dice, with different sample spaces. In the tetrahedron there are two numbers greater than 2, so there are half of the chances to get a number greater than 2 and in the hexahedron there are four numbers greater than 2 which corresponds to more than half the chances of getting one of these numbers. Although a significant number of participants evaluate the game as unfair, their justifications do not show an understanding of the proportional reasoning necessary for a coherent analysis of the situation, because they based their assessment on comparing absolute values (quantity of numbers greater than 2 in each dice) without establishing a relation with the total of each dice faces (proportional relation). For example, they stated:

C3 It is not fair because there are fewer numbers here than there. Here there is more possibility of getting numbers because it can come out $6,5 \ldots$ and here you can only go to 4 .

A5 It is unfair because Daniele has more scores than Priscila. This one has more advantage because it has more numbers greater than 2: 3, 4, 5 and 6 . This one only has up to 4 and higher than 2 only 3 and 4.

The children and adults seemed to be at the Subjective level of probabilistic thinking, as they compared probabilities of an event in two different sample spaces but based their justifications on various subjective or numerical judgments, without considering the proportional condition required by the situation. When asked how to make the game fair, the participants idea is that the use of different types of artefacts is an unfair condition, as it gives different chances for the players to win, so they proposed: (i) players' use of the same types of artefacts (tetrahedron, hexahedron or octahedron); and (ii) changes in the dice with a greater number of faces to try to match the numbers in all dice ("delete" numbers to be "equal" to others).

In summary, the adults and children's answers regarding how to make the game fair, also evidenced coherent and mistaken shared ideas about the fairness of the games, which are synthetized in Table 2. In general, the participants considered a game fair when the players have an equal chance of winning, but there was also the understanding that a game would be fair even with different chances for players to win. Luck was used as an argument associated with uncertainty of the results, and some students also resorted to the rules present in the games, such as Equal rules that were evidenced as a way to keep the game fair. In contrast, rules that seemed unequal to students, for example, the use of different artifacts, was seen as unfair. These ideas may be associated with the misunderstanding of the concept of randomness, when participants focus only on the uncertainty and unpredictability of the results, failing to analyze the sample space, for example.

Table 2. Ideas of children and adults about fair and unfair

\begin{tabular}{llll}
\hline & FAIR GAME & & UNFAIR GAME \\
Coherent ideas & Mistaken ideas & Coherent ideas & Mistaken ideas \\
\hline Equal chances & Fair with different chances & Dishonest randomizers & Chances: absolute value of \\
Luck & Use of equal artefacts & Different chances & numbers \\
Fair and equal rules & Cluttered numbers/ out of & & Different rules \\
Equilibrium & sequence & & Consecutive or ordered \\
& & numbers \\
& & Influence of data format \\
\hline
\end{tabular}




\section{CONCLUSIONS}

This study analyzed the understanding of children and adults about fairness in games, considering basic elements necessary to the understanding of probability (Bryant \& Nunes, 2012). The participants' answers to the proposed games showed that the judgments made about the (un)fairness of the games depended on their understanding about randomness, sample space, and comparing probabilities. In general, it was observed that both the children and adults had misunderstandings about these elements, leading participants to present incorrrect justifications to assess the fairness of the games.

As the participants did not have formal access to probability instruction and the adults' answers did not differ from those of the children, this study indicates that, according to Fischbein (1987), there are concepts that maturity for which maturity does not account, requiring intervention through teaching to expand and consolidate learning in the field of Probability. These facts also seem to indicate that daily experiences of adults are not enough to properly develop their probabilistic reasoning.

Compared to the children, the adults presented a greater number of responses using nonmathematical arguments irrelevant to the situation. As pointed out by Kahneman and Frederick (2001), the participants tended to rely on their experiences to make casual intuitive judgments on probabilistic facts, showing little knowledge about how their judgment and its logical implications arise. It seems to us that the intuitive beliefs of children, and especially of adults, that result from their experiences during the interview or their life, may lead to erroneous judgments regarding probabilistic situations and wrong answers and arguments without mathematical consistency, often based on common sense and personal experiences.

In the games that explored aspects of understanding probability, the children, on a larger scale, associated the fairness of the game with equiprobability. That is, the equal chances of the players to win, was associated with the idea of justice as balance, as also observed in Cañizares et al. (2004), whose students perceived what a balance provides. In this study, however, it was not unanimous, as some participants considered that if the player had any chance of winning, even if the chances were different from the opponents, the game would be fair. This result was surprising, considering the lack of research on this issue.

The misunderstanding of random sequences, based on informal beliefs and experiences, was configured as a weakness that greatly influenced the incorrect responses of children and adults in relation to randomness and fairness, while the observation of unfair randomizers was emphasized to identify unfairness in the game, resulting on a substantially higher number of correct answers in unfair than in fair games. This may have resulted from the fair game involving an analysis of random sequences, which required an understanding of event independence that proved complex for students (Bryant \& Nunes, 2012; Jones, 2006). These results are close to Pratt's (2000) results, as it was observed that irregularity was related to randomness when some participants considered irregular sequences to be random (e.g., LOTO game). The impossibility of being controlled and the unpredictability are rooted in the uncertainty that is the essence of randomness (Paparistodemou et al., 2002), elements observed in the students' justifications of the present study.

Although the responses about the games involving the sample space were substantially more correct, this does not guarantee that students had a better understanding of the sample space than of the other elements explored. The success of the participants in the responses may occurred because they did not have to explore the entire sample spaces. Instead, they were able to make conclusions about the fairness of the games based on other analyses. Thus, this study could be expanded with new proposals that consider all the sample space and its relationship with fairness in games, allowing students to deeply explore the elements of the events, given the difficulties they evidence in elaborating an exhaustive list of the sample space, as it requires combinatorial reasoning. The new proposals for the tasks may also consider compound or simple events to be a focus of further analysis.

In this study, the comparison of probabilities, involving different sample spaces, was shown to be an element of great fragility in the understanding of children and adults, due to the absence of proportional reasoning to analyze situations, similar to the reported by Bryant and Nunes (2012) who argue that a great advantage of proportional relations is that they make it possible to compare probabilities of different events, with different sample spaces. Without this understanding, the participants were unable to consciously assess (un)fairness of the games that required the comparison of different sample spaces, as they used the comparison of absolute quantities rather than relative 
quantities. This result highlights the need for teaching interventions that promote the expansion of proportional reasoning, not only in probability but also in other subjects (Bryant \& Nunes, 2012). Proportionality is considered by BNCC (ME, 2017) as a fundamental topic that needs to be developed at school to broaden the mathematical thinking of students in studies involving operations with natural numbers, fractions, rational numbers, area, functions and probability. Thus, we believe that research with an interventional focus that maximizes this understanding is essential.

In summary, the results of this study pointed out that participants:

(i) Both children and adults have difficulty in assessing fairness in games, if and when this assessment depends on understanding randomness regarding the independence of events and, particularly, on comparing the probability of different sample spaces;

(ii) Found it easy to assess an unfair game when observing unfair randomizers;

(iii) Consider the "balance" present in situations involving the sample space as a condition of fairness, as well as rules and types of artefacts;

(iv) Associate fair play with equal chances for players to win, but they can also consider it fair when there is a chance of winning, even if it is not equiprobable;

(v) Beliefs, common sense and personal experiences, influence their arguments for assessing (un)fairness of a game;

(vi) Misjudgement about fairness in games is influenced by misunderstanding of cognitive demands of probability.

Although the sample size of this research does not allow generalizations, the study provides clues about children and adults' understanding about fairness of games and the relationships between fair and unfair game judgments, as well as their knowledge regarding randomness, sample space and the comparison of probabilities. Thus, it may contribute, particularly, to research about the teaching and learning of probability. The results also provide important contributions that may assist in planning proposals to promote the understanding of probability of children and adults. We suggest, in agreement with Cañizares et al (2004) and Watson and Moritz (2003), to include fairness in the teaching of probabilities.

Regarding the activities presented in this study, they have the potential to be used for the diagnosis of children and adults' understanding of the relationship between the necessary elements to understand probability and fairness in games, as well as to support possible teaching interventions to expand students' probabilistic thinking. Further research is needed, however, especially of an interventional nature, to investigate how children and adults develop understanding of probability, and the ways of teaching that enable the understanding of the independence of events (understanding of randomness) and the comparison of probabilities in different sample spaces, with a focus on the development of proportional reasoning. It is also important to conduct studies about the understanding of the sample space, which implies, to some extent, the development of combinatorial reasoning.

\section{REFERENCES}

Batanero, C., Chernoff, E. J., Engel, J., Lee, H. S., \& Sánchez, E. (2016). Research on teaching and learning probability. ICME-13 Topical Surveys. Springer. https://doi.org/10.1007/978-3-31931625-3_1

Bennett, D. J. (1998). Randomness. Harvard University Press.

Borovcnik, M. (2016)._Pensamento probabilístico e alfabetização em probabilidade no contexto do risco. [Probabilistic thinking and probability literacy in the context of risk] Educação Matemática Pesquisa, 18(3), 1491-1516. https://revistas.pucsp.br/index.php/emp/article/view/31495

Bryant, P. \& Nunes, T. (2012). Children's understanding of probability: A literature review. Nuffield Foundation.

http://www.nuffieldfoundation.org/sites/default/files/files/Nuffield_CuP_FULL_REPORTv_FIN AL.pdf

Campos, T. M. \& Pietropaolo, R. C. (2013). Um estudo sobre os conhecimentos necessários ao professor para ensinar noções concernentes à probabilidade nos anos iniciais. [A study on teacher's knowledge to teach probability notions at the early years]. In R. Borba \& C. Monteiro (Orgs.), Processos de ensino e aprendizagem em Educação Matemática (pp. 55-91). UFPE. 
Cañizares, M. J., Batanero, C., Serrano, L., \& Ortiz, J. J. (2004). Children's understanding of fair games. In M. A. Mariotti (Ed.), European research in mathematics education III: Proceedings of 3rd Conference of the European Society for Research in Mathematics Education. CERME. http://www.mathematik.tu-ortmund.de/ erme/CERME3/Groups/TG5/TG5_canizares_cerme3.pdf

Carraher, T. N. (1998). O método clínico usando os exames de Piaget [The clinical method using Piaget's exams] (5th ed.). Cortez.

Chiesi, F., \& Primi, C. (2009). Recency effects in primary-age children and college studants. International Electronic Journal of Mathematics Education, 4(3), 149-164.

Fischbein, E. (1987). Intuition in science and mathematics: An educational approach. D. Reidel.

Franklin, C., Kader, G., Mewborn, D., Moreno, J., Peck, R., Perry, M., \& Schaeffer, R. (2007). Guidelines for assessment and instruction in statistics education report: A Pre-K-12 curriculum framework. American Statistical Association.

Jones, G. A (2006). The challenges of teaching probability in school. Griffith University. https://www.mathunion.org/fileadmin/ICMI/files/Digital_Library/ICMEs/Bulletin_Jones.pdf

Jones, G. A., Langrall, C. W., Thornton, C. A., \& Mogill, A. T. (1997). A framework for assessing young children's thinking in probability. Educational Studies in Mathematics, 32, 101-125. https://doi.org/10.1023/A:1002981520728

Jones, J. L. \& Tarr, J. E. (2007). An examination of the levels of cognitive demand required by probability tasks in middle grade mathematics textbooks. Statistics Education Research Journal, 6(2), 4-27. http://www.stat.auckland.ac.nz/serj

Lidster, S. T., Watson, J. M., Collis, K. F. \& Pereira-Mendoza, L. (1996). The relationship of the concept of fair to the construction of probabilistic understanding. In P. C. Clarkson (Ed.), Proceedings of the 19th Annual Conference of the Mathematics Education Research Group of Australasia (pp. 352-359). MERGA.

Ministério da Educação. (2017). Base Nacional Comum Curricular [BNCC]. Ministério da Educação, Secretaria da Educação Básica.

National Council of Teachers of Mathematics. (2000). Principles and standards for school mathematics. National Council of Teachers of Mathematics.

Paparistodemou, E., Noss, R., \& Pratt, D. (2002). Exploring sample space: Developing young children's knowledge of randomness. In B. Phillips (Ed.), Proceedings of the 6th International Conference on Teaching of Statistics (ICOTS 6), Cape Town, South Africa, July 7-12. International Statistical Institute. https://iase-web.org/documents/papers/icots6/2a3_papa.pdf?1402524960

Pratt, D. (2000). Making sense of the total of two dice. Journal for Research in Mathematics Education, 31(5), 602-625. https://doi.org/10.2307/749889

Sharma, S. (2016). Probability from a socio-cultural perspective. Statistics Education Research Journal, 15(2), 126-144. https://doi.org/10.52041/serj.v15i2.244

Vidakovic, D., Berenson, S., \& Brandsma, J. (1998). Children's intuitions of probabilistic concepts emerging from fair play. In L. Pereira-Mendoza, L. S. Kea, T. W. Kee, \& W. Wong (Eds.), Proceedings of the 5th International Conference on Teaching Statistics, Singapore, June 21-26 (Vol. 1, pp. 67-73). IASE. https://iase-web.org/documents/papers/icots5/Topic1e.pdf?1402524956

Watson, J. M., \& Moritz, J. B. (2003). Fairness of dice: A longitudinal study of students' beliefs and strategies for making judgments. Journal for Research in Mathematics Education, 34 (4), 270-303. https://doi.org/10.2307/30034785

Wolcott, H. (2009). Writing up qualitative research (3rd ed.). SAGE.

ANA HENRIQUES

Instituto de Educação, Universidade de Lisboa Alameda da Universidade 1649-013 Lisboa, Portugal 